\title{
Total Abdominal Hysterectomy
}

National Cancer Institute

\section{Source}

National Cancer Institute. Total Abdominal Hysterectomy. NCI Thesaurus. Code C51695.

Surgical removal of all of the uterus, via an abdominal approach. 